\title{
020 PP ENABLING COLLABORATIVE HEALTH RESEARCH: A QUALITATIVE LONGITUDINAL STUDY OF A LARGE-SCALE CO-PRODUCTION PROGRAMME
}

R Kislov. Alliance Manchester Business School, University of Manchester, UK

10.1136/bmjopen-2017-016492.38

Objective: The study aims to address the following research question: How do the co-production activities and structures develop over time?

Design: Qualitative longitudinal case study comprised of five rounds of semi-structured interviews, observation and documentary analysis conducted in 2009-2016.

Setting: A large-scale government-funded collaborative research partnership established in 2008 aiming to produce and implement high-quality applied health research in collaboration with local National Health Service (NHS) organisations.

Participants: 102 research participants $(4$ applied health researchers; 7 management researchers, 11 clinical researchers; 8 facilitators; 4 research managers; 4 commissioners; 15 medical managers; 23 nurses; 21 general practitioners; 5 auxiliary health professionals) selected due to their participation in the co-production of applied health research and its implementation.

Results: The following four trends in the evolution of co-production have been identified: (1) shift from the initial separation of 'research' and 'implementation' towards their integration; (2) shift from a number of bounded silos towards enabling the 'cross-cutting' way of working; (3) shift from a relatively rigid structure towards a flexible framework that can be modified depending on the specific project needs; (4) shift from individual knowledge brokering roles towards collective brokering performed by multiprofessional teams.

Conclusions: We explain these trends by an increasing organisational emphasis on the iterative processes of critical reflection and reflexivity which draw on multiple sources of actionable knowledge in order to adjust the co-production activities to the constantly changing internal and external context. 